\title{
Emergence of Smooth Pursuit using Chaos
}

\author{
Boris Duran, Giulio Sandini, Giorgio Metta \\ Italian Institute of Technology - University of Genova \\ Emails: boris@unige.it, giulio.sandini@iit.it, pasa@liralab.it
}

\begin{abstract}
The task of tracking an object has been fully studied and many solutions presented before. However, it is a perfect test bed for the study of a novel model using Coupled Chaos Systems. Once an object appears in front of a camera, we demonstrate that the visual input is enough for the self-organization of the torques applied to each of the axes controlling the motion of a simulated eye. No learning or specific coding of the task is needed beforehand, which results in a very fast adaptation to perturbations.
\end{abstract}

\section{Introduction}

The research in modern humanoid robotics has a history of approximately 30 years when the Bioengineering group of Waseda University started the WABOT project. Some years later, Honda started also their research, the result of which is one of the state-ofthe-art humanoids of our time, ASIMO. Most of today's humanoid platforms follow a, more or less, 50year-old tradition of Control Theory that started with Industrial Automation at the beginning of the 1960s. Control Theory gives us different tools for designing and evaluating the algorithms that will realize a desired motion or force application [1]. It is in this point where the problems start for the humanoids of the future.

Mechanical manipulators can be very precise given detailed descriptions of the task to fulfill, and of course, describing the manipulator completely as well. In an industrial environment this is not a problem; we are able to specify within centimeters, distances, area of motion, speed and acceleration of different links, force and torques, etc. But what happens when we want to move from this fixed framework? If we want to design and build systems that move and act in the same kind of environment that we humans move and act, we will have to move from the Classical Control Theory approach. A more adaptive and flexible theory is needed when thinking of "controlling" a device that is supposed to move within an ever-changing environment.

The study of Nonlinear Dynamics and Chaos has also a long history; however, real applications that make direct use of Chaos Theory have not been fully developed. The purpose of this research is to demonstrate the feasibility of using Coupled Chaotic Systems in a more realistic application by taking the model of behavior emergence introduced by Kuniyoshi et al. [2] within the area of Humanoid Robotics.

The task of Smooth Pursuit has been solved in many different and more accurate ways than the one presented here. However, this task presents a very simple and attractive challenge to use as test bed for Coupled Chaotic Systems. Another interesting point to be considered is the notion of emergence and selforganization that characterize these systems. And it is exactly in this point where a link between Chaos and Biological systems can be found. It would not be strange to think that this reactive-emergent kind of behavior generated by Coupled Chaos has its counterpart in the Biological Nervous System.

Walter Freeman and colleagues have done an extensive research on the dynamics found in EEG waves from the mammalian olfactory system [8]. He has demonstrated the existence of chaotic dynamics during perception at a mesoscopic level, which refers to the level in between the analysis of single neurons (microscopic) and the activity of whole brain areas (macroscopic). Since it has been shown that nature uses chaos to self-organize the information coming from our senses, we may assume that chaos is also used to organize our muscular responses. With this in mind a simple experiment of self-organizing behavior is studied in this project by using Coupled Chaotic Systems.

The next section describes the basics of Coupled Chaotic Systems together with the model of behavior emergence proposed in [2]. Next, a description of the setup used for Smooth Pursuit is presented together with the quantitative analysis of the experiment; and, 
finally, we present the conclusions and guidelines for future work.

\section{Coupled Chaotic Systems}

A network of elements which activation is defined by a chaotic map receives the name of Coupled Chaotic Systems. Depending on the level of interaction among their elements, it is possible to classify them in systems of local or global interaction.

\subsection{Coupled Map Lattices}

Coupled Map Lattices (CML) was introduced by Kaneko in the middle of the 80's as an alternative for the study of spatiotemporal chaos [4]. In short, this kind of dynamical systems use discrete partial difference equations to study the evolution of a process described by discrete steps in space and time but with continuous states. Equation (1) describes the dynamics of CML.

$$
\begin{gathered}
x_{n}^{i}=(1-\varepsilon) f\left(x_{n-1}^{i}\right)+\frac{\varepsilon}{2}\left\{f\left(x_{n-1}^{i+1}\right)+f\left(x_{n-1}^{i-1}\right)\right\} \\
f(x)=1-\alpha \cdot x^{2}
\end{gathered}
$$

Where $x_{n}{ }^{i}$ is a variable at discrete time step $n$ and a lattice point $i . x$ represents a set of field variables which could be temperature, position measurements, velocities, etc. There are two parameters: $\alpha$ controlling the level of chaoticity of the system and $\varepsilon$ controlling the coupling level among neighbor elements.

\subsection{Globally Coupled Maps}

These kinds of maps were also introduced by Kaneko and represent a network of chaotic elements with interactions among all of them. While CML interact with specific points within the lattice, each of the nodes in a Globally Coupled Map (GCM) interact with all the others. Due to the chaotic nature of the system, specified by $\alpha$, it is possible to see one of the main properties of chaotic systems: two slightly different initial conditions amplify its difference through time. On the other hand, $\varepsilon$ tries to synchronize the activations of all these chaotic elements by coupling them. In between these two states of complete chaos and complete synchronization, interesting states emerge like the formation of clusters oscillating in different phases and amplitudes.

$$
x_{n}^{i}=(1-\varepsilon) f\left(x_{n-1}^{i}\right)+\frac{\varepsilon}{N} \sum_{j=1}^{N} f\left(x_{n-1}^{j}\right)
$$

Both of these categories have been deeply studied during the last two decades; trying to describe them both qualitatively and quantitatively. The effects of varying both, chaoticity and coupling factor, in standalone CML and GCM systems were studied meticulously by Kaneko's group in the late 90's [5, 6]. Approximate phase diagrams were sketched covering all the spectrum of synchronization among the interacting chaotic elements of a network.

\subsection{Coupled Chaotic Fields}

The model used in this project is based on the approach followed by Kuniyoshi and Suzuki [2]. The main idea behind this model is to make use of all those interesting states mentioned before that emerge when coupling chaotic elements; and, in this case, the sensory information is modified by the environment. It is being used both, the local interaction (CML) and the global interaction (GCM). The system is depicted in the following diagram.

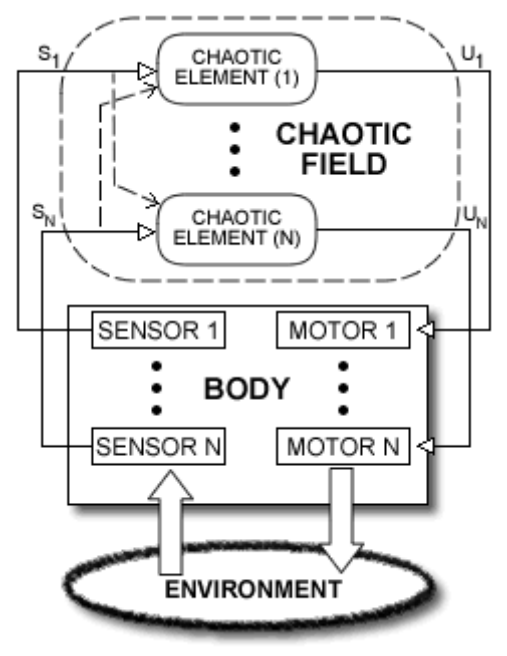

Figure 1. Body and environment interaction through Chaotic Fields.

Each one of the blocks containing "Chaotic" elements and their relationship constitute the core of the system and it is defined by the following equations. The function $\mathrm{f}$ represents the Logistic Map mentioned in the previous section, Equation 2.

$$
u_{n}^{i}=f\left\{s_{n-1}^{i}+\varepsilon_{1}\left(\bar{s}_{n-1}-s_{n-1}^{i}\right)+\varepsilon_{2}\left(\frac{s_{n-1}^{i+1}+s_{n-1}^{i-1}}{2}-s_{n-1}^{i}\right)\right\}
$$




$$
\begin{aligned}
& m_{n}^{i}=G_{u}\left(u_{n}^{i}+O_{u}\right) \\
& s_{n}^{i}=G_{r}\left(r_{n}^{i}+O_{r}\right)
\end{aligned}
$$

Where $m$ is the torque applied to each servo, $s$ and $u$ are inputs and outputs respectively of the chaotic field block, and $r$ is the raw value coming from the plant. Finally, $G_{u}, G_{r}, O_{u}$, and $O_{r}$ are gains and offsets of the sensors and servos in the body of the plant; these values are applied in the same magnitude to all the elements of the system. They were fixed to $G_{u}=1.5$, $G_{r}=1.0, O_{u}=-0.5$, and $O_{r}=-0.2$.

\section{A virtual eye}

To simulate the dynamics of an artificial eye, a virtual environment named Webots has been used [9]. This software is based in the Open Dynamics Engine libraries for reasonably accurate physics simulation such as the effect of gravity and friction. The time step for the simulations was fixed to 64 milliseconds and the experiments were done without the influence of gravity and with a minimum value of friction.

The virtual eye was created using two rotational servos, one perpendicular to the other in order to simulate the "yaw" and "pitch" motions of a real eye. See Figure 2. Each servo is modeled also by a spring and a damper, trying to replicate also the physical characteristics of real muscles. These two servos are actuating the virtual eye as the motion created by the main four muscles in biological eyes.
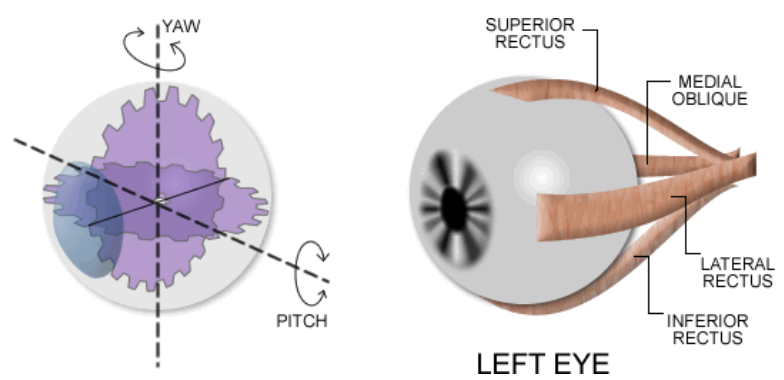

Figure 2. Biological eye and its virtual
counterpart for the experiment.

A virtual camera was used to simulate the Field of View (FOV) of the eye. The width and height were fixed to $32 \times 32$ pixels with a FOV of 0.5 radians. The object to be tracked was simulated by a black circular shape moving on a white background, see Figure 3. Even though the camera was set for tracking grey variations, it is also possible to work with color objects. For the sake of simplicity it was chosen the black and white camera since the purpose of the experiment is not the vision problem, in other words, it is assumed that we obtain values of saliencies from other visual components. One of these saliencies is our black object moving on a white screen. Even though the trajectory followed by the object is circular all the time, it accelerates and slows downs several times to have a basic test for the robustness of the system.

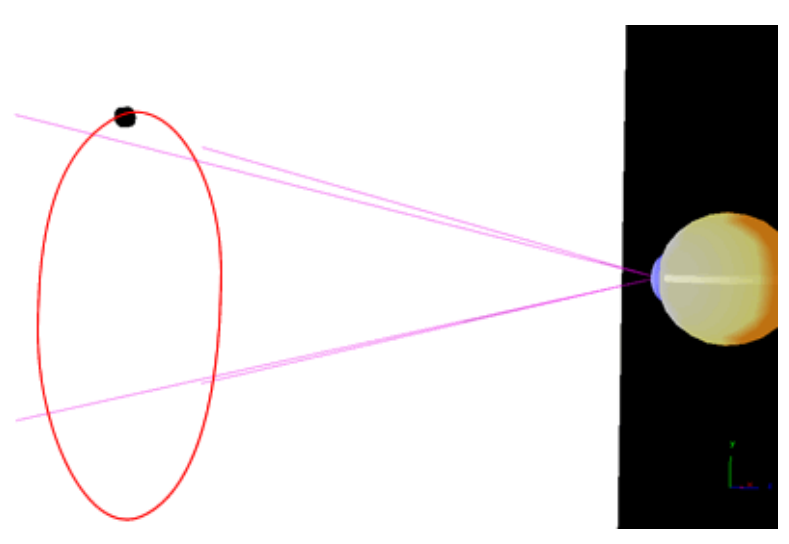

Figure 3. Screenshot of the virtual setup.

The input to the system is given by the difference between the center of the observed object within the field of view and the position of the center of the eye, for both the vertical and horizontal motions, Figure 4. The outputs from the chaotic field are directly fed to the motors.

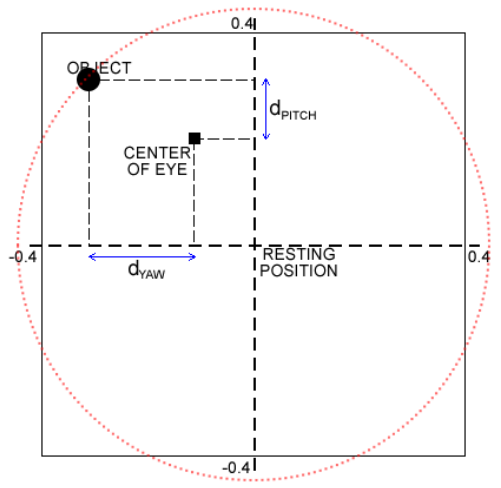
Figure 4. Geometric description for inputs to
the chaotic system.

The trajectory followed by our virtual eye can be observed in the following plot. The red circle marks the position of the eye at the beginning of the simulation. As we can see, the first reaction, once the object has entered into the Field of View of the eye, is to move towards the object. There is a small period of oscillations until it reaches the "smooth" pursuit state. 


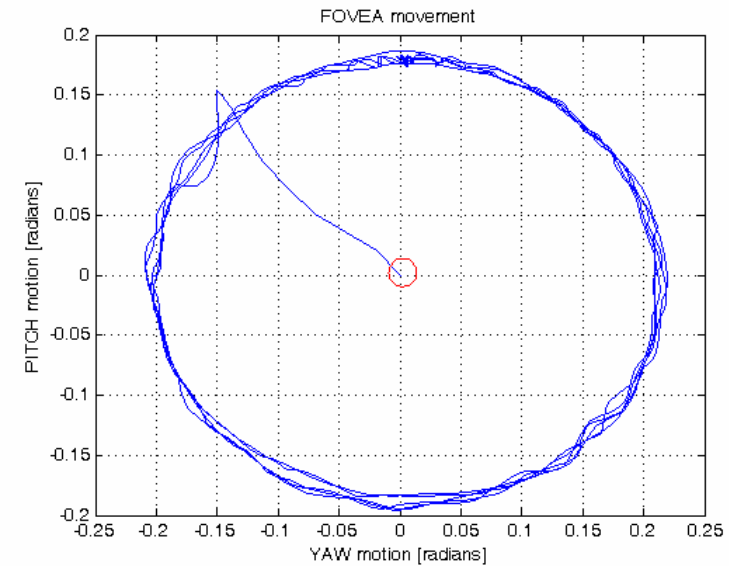

Figure 5. Trajectory of the center of the eye $(\alpha=1.95, \varepsilon=0.1$ ).

Even though the "eye" is trying to follow the object as smoothly as possible, the system keeps a, more or less, constant error through all the simulation time, see Figure 6. This error is related to the position of the object and seems to be directly influenced by the physical characteristic of the hardware.

Another important information from this plot is the small time elapsed until it reaches this "steady" state. In less than one second, the system adapts to the recent change in the environment. Arrows indicate the moments where the object slows down to change its direction of motion. In these moments both motors, the YAW and PITCH motors, have the opportunity to couple as much as possible; whereas when the object is moving at its maximum speed, the motors are completely out of phase.

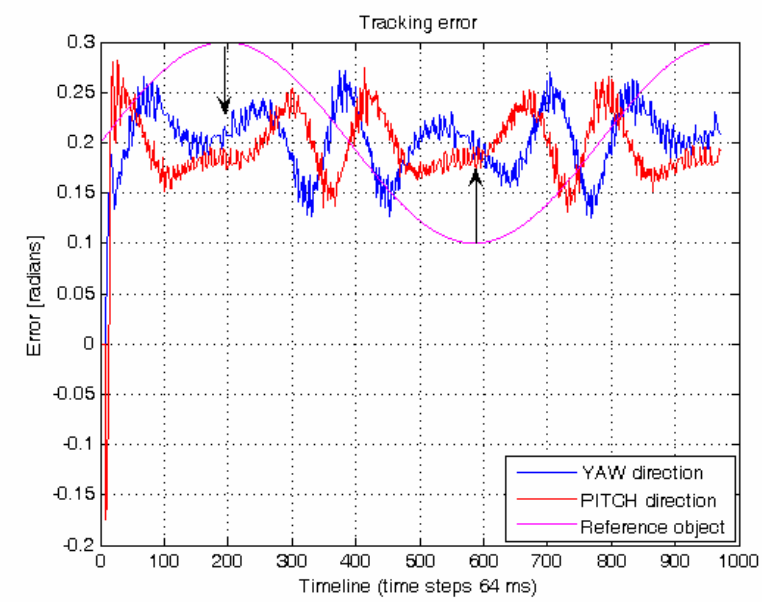

Figure 6. Tracking error. Arrows indicate the moments of change of direction of the object.
The "smooth pursuit"-kind of motion was tested using different values of $\alpha$ (chaoticity factor) and $\varepsilon$ (coupling factor). However when either $\varepsilon$ was greater than 0.35 or $\alpha$ lower than 0.1 the system performs inconsistent movements, sometimes trying to follow the object and sometimes trying to escape from it. Figure 7 shows the behavior of the "eye" for $\alpha=0.1$ and $\varepsilon=0.2$.

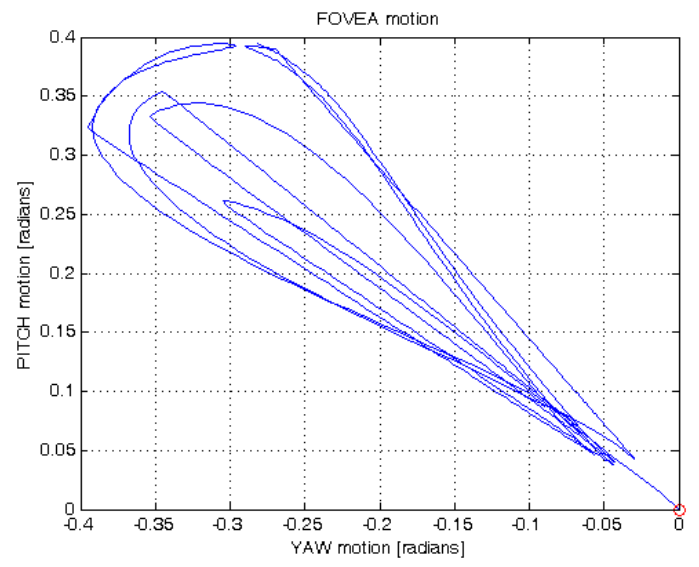

Figure 7. Trajectory of the center of the eye $(\alpha=0.1, \varepsilon=0.2)$.

A deep study on the effects of varying these two parameters, $\alpha$ and $\varepsilon$, would be very difficult because of the local and global interaction of elements at the same time; moreover, every observation would change from time to time due to the ever changing influence of the environment.

\section{Conclusions and Future Work}

A very simple experiment for demonstrating the feasibility of applying Coupled Chaos Systems in Humanoid Robotics has been shown in this project. Tracking an object moving in front of a camera has been solved in several ways previously, from using very simple trigonometric solutions to advanced control algorithms. However, this task was simple enough to use it in the emergence of a reactive behavior that could have a better understanding in Neural Sciences.

According to neurosciences, all behavior is mediated by the Central Nervous System (brain and spinal cord) which is separated but functionally interconnected with the Peripheral Nervous System (continuous stream of sensory information about the environment). Simple put, the major difference between voluntary and reflexive movements is the intervention or not of the Central Nervous System [3]. 
In practice, it is not possible to separate the modulation signals coming from the brain into the muscles of our eyes. But according to the results of this experiment, we could speculate that visual tracking is just a reactive behavior given a saliency in our visual field. These saliencies are the necessary modulations given by our Central Nervous System and its areas of emotions, experiences, needs, etc.

Future work involves the coordination of motion with two eyes and finally the emergence of coordinated motion between eyes and head. The simulation environment saves a lot of time and resources for this type of experiments; however, a great amount of that saved time is dedicated only to tune the physical parameters in these simulated environments. Most of the software for robotics simulation still creates instabilities influencing the way the algorithms are supposed to work. Therefore, an $\mathrm{iCub}$ head from the RobotCub project [7] is being developed to test this and future experiments in a real environment.

\section{References}

[1] J. Craig, Introduction to Robotics: Mechanics and Control, Pearson Education, 2004.

[2] Y. Kuniyoshi, S. Suzuki, "Dynamic Emergence and Adaptation of Behavior through Embodiment as a Coupled Chaotic Field", Proceedings IEEE/RSJ, International Conference on Intelligent Robots and Systems, Sendai Japan, 2004, pp. 2042-2049.

[3] E.R. Kandel, J.H. Schwartz, T.M. Jessell, Principles od Nerual Sciences, McGraw-Hill, 2000.

[4] K. Kaneko, I. Tsuda, Complex Systems: Chaos and Beyond, Springer-Verlag, 2001.

[5] K. Kaneko, "Pattern Dynamics in Spatiotemporal Chaos", Physica D34, Elsevier Science Publishers, Holland, 1989, pp. $1-41$.

[6] K. Kaneko, "Clustering, Coding, Switching, Hierarchical Ordering and Control in a Network of Chaotic Elements", Physica D41, Elsevier Science Publishers, Holland, 1990, pp. 137-172.

[7] RobotCub Project, http://www.robotcub.org.

[8] W. Freeman, Mass Action in the Nervous System, Academic Press Inc., New York, 2004.

[9] Cyberbotics Webots, http://www.cyberbotics.com. 